\title{
The Fully Lagrangian Approach to the Analysis Of Particle/Droplet Dynamics: Implementation into ANSYS Fluent and Application to Gasoline Sprays
}

\author{
Timur S. Zaripov, ${ }^{1,2, *}$ Artur K. Gilfanov, ${ }^{2}$ Steven M. Begg, ${ }^{1}$ \\ Oyuna Rybdylova, ${ }^{1}$ Sergei S. Sazhin, ${ }^{1}$ E Morgan R. Heikal ${ }^{1}$ \\ ${ }^{1}$ Advanced Engineering Centre, School of Computing, Engineering \\ and \\ Mathematics, University of Brighton, Brighton, BN2 4GJ, UK \\ ${ }^{2}$ Kazan (Volga region) Federal University, Kazan, Russian Federation.
}

${ }^{*}$ Address all correspondence to Timur S. Zaripov

E-mail: zaript@gmail.com, S.Sazhin@brighton.ac.uk

Original Manuscript Submitted: 03/06/2017; Final Draft Received: 03/06/2017

The fully Lagrangian approach (FLA) to the calculation of the number density of inertial particles in dilute gas-particle flows is implemented into the CFD code ANSYS Fluent. The new version of ANSYS Fluent is applied to modelling dilute gas-particle flow around a cylinder and liquid droplets in a gasoline fuel spray. In a steady-state case, the predictions of the FLA for the flow around a cylinder and those based on the equilibrium Eulerian method (EE) are almost identical for small Stokes number, $\mathrm{Stk}$, and small Reynolds number, $\mathrm{Re},(\mathrm{Re}=1, \mathrm{Stk}=0.05)$. For the larger values of these numbers $(\mathrm{Re}=10,100 ; \mathrm{Stk}=0.1,0.2)$ the FLA predicts higher values of the gradients of particle number densities in front of the cylinder compared with the ones predicted by the EE. For transient flows $(\mathrm{Re}=200)$, both methods predict high values of the number densities between the regions of high vorticity and very low values in the vortex cores. For Stk $\geq 0.1$ the maximal values predicted by FLA are shown to be several orders of magnitude higher than those predicted by the EE. An application of FLA to a direct injection gasoline fuel spray has focused on the calculation of the number densities of droplets. Results show good qualitative agreement between the numerical simulation and experimental observations. It is shown that small droplets with diameters $d_{\mathrm{p}}=2 \mu \mathrm{m}$ tend to accumulate in the regions of trajectory intersections more readily, when compared with larger droplets $\left(d_{\mathrm{p}}=10 \mu \mathrm{m}, d_{\mathrm{p}}=20 \mu \mathrm{m}\right)$. This leads to the prediction of the regions of high number densities of small droplets.

KEY WORDS: Gas-particle flow Particle number densities Eulerian approach Fully Lagrangian approach Gasoline fuel sprays. 


\section{INTRODUCTION}

The analysis of particle/droplet dynamics is commonly based on the Eulerian-Lagrangian approach in which the Eulerian approach is used for the analysis of the carrier phase dynamics while the Lagrangian approach is used for the analysis of the dispersed phase. This approach is used in all modern CFD codes, including VECTIS (Sazhina et al., 2000) and ANSYS Fluent (Rybdylova et al., 2016), alongside variants of the EulerianEulerian approach. A comparative assessment of the variety of methods under these two approaches is given by Kolakaluri et al. (2014).

Alternative methods for the calculation of particle number densities were suggested by Fernandez de la Mora and Rosner (1981) and Osiptsov (1997). These are known as the fully Lagrangian approaches (methods) and both of these are focused on the calculation of the evolution of particle number densities along particle trajectories. A detailed comparison between these approaches was performed by Healy and Young (2005), who concluded that Osiptsov's method has clear advantages over the one suggested by Fernandez de la Mora and Rosner. The method suggested by Osiptsov (1997, 2000), hereafter referred to as the fully Lagrangian approach or FLA, is based on the particle continuity equation in the Lagrangian form and predicts particle number densities along trajectories by solving the equations for the components of the Jacobi matrix of the Eulerian-Lagrangian transformation. The method allows us to perform accurate calculations in the regions of high number density gradients and in the regions where intersections of the particle trajectories (folds) occur. Also, it was shown by Healy and Young (2005), that FLA is approximately 20 times more efficient than the traditional Lagrangian approach in the two-dimensional case and even more so in the threedimensional case.

Although the fully Lagrangian approach has been discussed in the literature, it has never been applied in the field of engineering modelling to the best of our knowledge. In this work we intend to demonstrate the applicability of the FLA to two typical modelling tasks that require calculation of particle number densities: dilute gas-particle flow around a blunt body (cylinder), that is encountered in aerosol filtering (Friedlander, 2000), and the modelling of a hollow-cone gasoline fuel spray (Heywood, 1988). This applicability will be investigated through the implementation of the fully Lagrangian approach into the commercial CFD code ANSYS Fluent using User Defined Functions (UDF), that provide an access to flow variables during computations.

Mathematical models of the gas-particle flow and details of the implementation are described in sections 2 and 3. In section 4 the results of application of the new customised version of ANSYS Fluent to the analysis of a dilute gas-particle flow around an infinitely long circular cylinder are presented. The predictions of this code are compared to the predictions based on the equilibrium Eulerian method, proposed by Ferry and Balachandar (2001). Results of the application of this version of ANSYS Fluent to 
the analysis of the results of experimental observations of a gasoline fuel spray, at representative fuel injection conditions, are presented in section 5. The numerical results are compared with experimental data where appropriate. The main results of the paper are summarised in section 6 .

\section{MATHEMATICAL MODELS OF THE CARRIER AND DISPERSED PHASES}

\subsection{Gas (carrier phase) flow}

The carrier phase is modelled as an incompressible or compressible viscous flow of gas described by the mass continuity and Navier-Stokes equations. In the case of an incompressible flow these equations are presented as

$$
\begin{aligned}
\nabla \cdot \mathbf{u} & =0 \\
\rho \frac{\partial \mathbf{u}}{\partial t}+\rho(\mathbf{u} \cdot \nabla) \mathbf{u} & =-\nabla p+\mu \nabla^{2} \mathbf{u},
\end{aligned}
$$

where $\rho$ is the gas density, $\mathbf{u}$ is the gas velocity, $p$ is the static pressure, $\mu$ is the gas dynamic viscosity.

Flow Reynolds number is defined as

$$
\operatorname{Re}=\frac{\rho L U}{\mu}
$$

where $L$ and $U$ are characteristic length and velocity scales.

\subsection{Particles (droplets) in a gas flow}

Particles (or droplets) are assumed to be spherical and the Stokes drag law is valid. This is justified for the particle Reynolds numbers:

$$
\operatorname{Re}_{\mathrm{p}}=\frac{\rho d_{\mathrm{p}}|\mathbf{u}-\mathbf{v}|}{\mu} \leq 1
$$

where $\mathbf{v}$ is the particle velocity, and $d_{\mathrm{p}}$ is the particle diameter. For higher $\mathrm{Re}_{\mathrm{p}}$ a correction should be included in the drag law, such as the Oseen, Massley, Sternin or Klyachko corrections (Nigmatulin, 1990).

We assume that the density of particle material $\rho_{p} \gg \rho$. In this case buoyancy, added-mass and Basset-Boussinesq forces can be ignored. Also, particles are assumed to be large enough to allow us to ignore Brownian forces. In this case the equations of 
particle motion are written as

$$
\begin{aligned}
& \frac{d \mathbf{x}}{d t}=\mathbf{v}, \\
& \frac{d \mathbf{v}}{d t}=\beta(\mathbf{u}-\mathbf{v}),
\end{aligned}
$$

where $\mathbf{x}$ and $\mathbf{v}$ are particle position vector and velocity in Eulerian coordinates, $\beta=\tau_{\mathrm{p}}^{-1}$ is the drag force coefficient.

Particle response time $\tau_{\mathrm{p}}$ is estimated as

$$
\tau_{\mathrm{p}}=\frac{\rho_{\mathrm{p}} d_{\mathrm{p}}^{2}}{18 \mu}
$$

The ratio of $\tau_{p}$ and the characteristic flow time $\tau_{\mathrm{f}}$ is known as the Stokes number

$$
\operatorname{Stk}=\frac{\tau_{\mathrm{p}}}{\tau_{\mathrm{f}}} .
$$

\subsection{Fully Lagrangian method}

In contrast to the conventional Lagrangian method, that focuses on the analysis of trajectories of individual particles, the fully Lagrangian method describes the evolution of particle number densities in time and space. The expression for the normalised number density $n_{\mathrm{p}}$ of particles along the particle trajectory, as inferred from the mass conservation, can be written as (Osiptsov, 1997)

$$
n_{\mathrm{p}}=\frac{n_{0}}{|J|},
$$

where $n_{0}$ is the initial particle number density, $|J|$ is the absolute value of the Jacobian of the Eulerian-Lagrangian transformation (absolute value of the determinant of the Jacobi matrix). Both $n_{\mathrm{p}}$ and $J$ are functions of $t$ and Lagrangian coordinates $\left\{\mathbf{x}_{0}, t_{0}\right\}$, defined by the starting point of the trajectory.

The components of the Jacobi matrix are defined as

$$
J_{i j}=\left(\frac{\partial x_{i}}{\partial x_{j 0}}\right)
$$

where $x_{i}$ and $x_{j 0}$ are components of $\mathbf{x}$ and $\mathbf{x}_{0}$ respectively.

Differentiating both parts of Eq. (5) with respect to $x_{j 0}$ we obtain

$$
\frac{\partial \omega_{i j}}{\partial t}=\beta\left(\frac{\partial u_{i}}{\partial x_{j 0}}-\omega_{i j}\right)
$$


where

$$
\omega_{i j}=\frac{\partial J_{i j}}{\partial t}=\frac{\partial v_{i}}{\partial x_{j 0}} .
$$

Eqs. (10) and (9) are rewritten as (Osiptsov, 1997)

$$
\begin{aligned}
\frac{\partial J_{i j}}{\partial t} & =\omega_{i j} \\
\frac{\partial \omega_{i j}}{\partial t} & =\beta\left(\sum_{k}\left(J_{k j} \frac{\partial u_{i}}{\partial x_{k}}\right)-\omega_{i j}\right) .
\end{aligned}
$$

In the two-dimensional case $(i, j, k=1,2)$, Eqs. (11) and (12) is the system of 8 ordinary differential equations with respect to time along the particle trajectories, calculated based on Eqs. (4)-(5). The values of $n_{\mathrm{p}}$ along these trajectories are inferred from Eqs. (8).

At the start of the trajectory, the Lagrangian and Eulerian coordinates are equal, hence, initial conditions for the $J_{i j}$ are always the same: $\left(J_{11}\right)_{0}=\left(J_{22}\right)_{0}=1,\left(J_{12}\right)_{0}=$ $\left(J_{21}\right)_{0}=0$. Initial values of the $\omega_{i j}$ are inferred from the velocity field of the carrier phase at the starting point by assuming $x_{j 0}=x_{j}$ in Eq. (10).

The fully Lagrangian method is easy to implement and computationally efficient. The results obtained using FLA do not depend on the number of simulated particles, unless interpolation of the number density values on the Eulerian grid is required.

\subsection{Equilibrium Eulerian method}

For the flow around a cylinder, the predictions of the fully Lagrangian approach will be compared with the predictions of the equilibrium Eulerian approach. In the latter approach, the average particle velocity is expressed as a function of the carrier phase velocity using an expansion over particle response time $\tau_{p}$ (Maxey, 1987). When $\tau_{p}=$ 0 , particle velocity $\mathbf{v}$ coincides with the velocity of the carrier phase $\mathbf{u}$. When $\tau_{p}$ is small relative to $\tau_{\mathrm{f}}, \mathbf{v}$ is estimated as

$$
\mathbf{v}=\mathbf{u}-\tau_{\mathrm{p}}\left(\frac{\partial \mathbf{u}}{\partial t}+\mathbf{u} \cdot \nabla \mathbf{u}\right)+O\left(\tau_{\mathrm{p}}^{2}\right)
$$

Eq. (13) allows us to obtain the values of $\mathbf{v}$ if the solution to Eqs. (1) is known.

The transport equation for the number density of monodisperse particles within this approach is written as (Friedlander, 2000):

$$
\frac{\partial n_{\mathrm{p}}}{\partial t}+\nabla \cdot\left(n_{\mathrm{p}} \mathbf{v}\right)=\nabla \cdot\left(D \nabla n_{\mathrm{p}}\right)
$$


where $D$ is the diffusion coefficient estimated as

$$
D=\frac{k_{\mathrm{B}} T}{3 \pi \mu d_{\mathrm{p}}}
$$

$k_{\mathrm{B}}$ is the Boltzmann constant and $T$ is the reference temperature.

The advantage of this method is that the particle velocity can be found without solving additional partial differential equations for the particle velocity field. This method was generalised by Ferry and Balachandar (2001) and it was shown that the first order approximation of particle velocity leads to sufficiently accurate results. The equilibrium Eulerian method is well known, easy to implement and computationally efficient, which makes it a good for comparison with FLA at low Stokes numbers. We will refer to the equilibrium Eulerian method as the Eulerian method.

\section{IMPLEMENTATION OF THE METHODS INTO ANSYS FLUENT}

\subsection{Fully Lagrangian method}

Eqs. (4)-(5) are solved using the varying order version of the Runge-Kutta method implemented in ANSYS Fluent (Cash and Karp, 1990). Eqs. (11)-(12) are solved at each point of a trajectory using the in-house implementation of the fourth order RungeKutta method. This solver is implemented in ANSYS Fluent via User Defined Functions (UDF). The DEFINE_DPM_UPDATE_SCALAR macros is used to execute the in-house code inside ANSYS Fluent. The initial conditions for $x_{i}, v_{i} J_{i j}, \omega_{i j}$ and $n_{\mathrm{p}}$ are specified using DEFINE_DPM_INJECTION_INIT macros.

During parallel executions, the solution domain is split into partitions (sub-domains) with one computational node responsible for each partition. As particles travel between partitions, they are assigned to new nodes. During this process, the values of all local and global variables in the relevant UDF code are lost. In order to keep the required values, the P_USER_REAL(p, i) macros was used.

\subsection{Equilibrium Eulerian method}

ANSYS Fluent allows users to solve the general transport equation for an arbitrary scalar function $\phi$

$$
\frac{\partial \rho \phi}{\partial t}+\nabla \cdot\left(\rho \mathbf{u}_{\phi} \phi\right)=\nabla \cdot(\Gamma \nabla \phi)+S_{\phi}
$$

where $\Gamma$ and $S_{\phi}$ are the diffusion coefficient and the source term for $\phi$, respectively.

Each term in Eq. (16) can be specified using UDF. For $\phi=n_{\mathrm{p}}, \rho=1, \Gamma=D$, $S_{\phi}=0$ and $\mathbf{u}_{\phi}=\mathbf{v}$, Eq. (16) reduces to Eq. (14). Eq. (14) was solved using Eq. (13) to calculate $\mathbf{v}$. 


\subsection{Verification of the results}

Wessel and Righi (1988) considered the motion of inertial particles with various Stokes numbers in a potential flow of gas around an infinitely long circular cylinder. They calculated normalised number densities of the particles deposited on the surface of the cylinder using the method of Fernandez de la Mora and Rosner (1981) (FMR). Both FMR and the fully Lagrangian approach (FLA) are based on the solution of the particle mass conservation equation along the particle pathlines (see Eq. (8)). The difference between these approaches lies in the methods used in solving this equation. In the FMR this equation is solved in the Eulerian space, while in the FLA it is transformed into Lagrangian form and solved in the Lagrangian space, allowing us to handle trajectory intersections that produce singularities in number densities. If no trajectory crossing occurs, as in the case considered by Wessel and Righi (1988), both methods produce similar results.

The number densities obtained by Wessel and Righi (1988) were compared with the ones predicted by the customised version of ANSYS Fluent with the the implementation of fully Lagrangian approach. The analytical expression for the potential flow of the gas was used. The plots of these densities, predicted by both approaches, versus angle $\theta$ are shown in Fig. 1. Solid curves show the results obtained using the FLA, symbols show the results presented by Wessel and Righi (1988). A good agreement between the predictions of both approaches can be clearly seen.

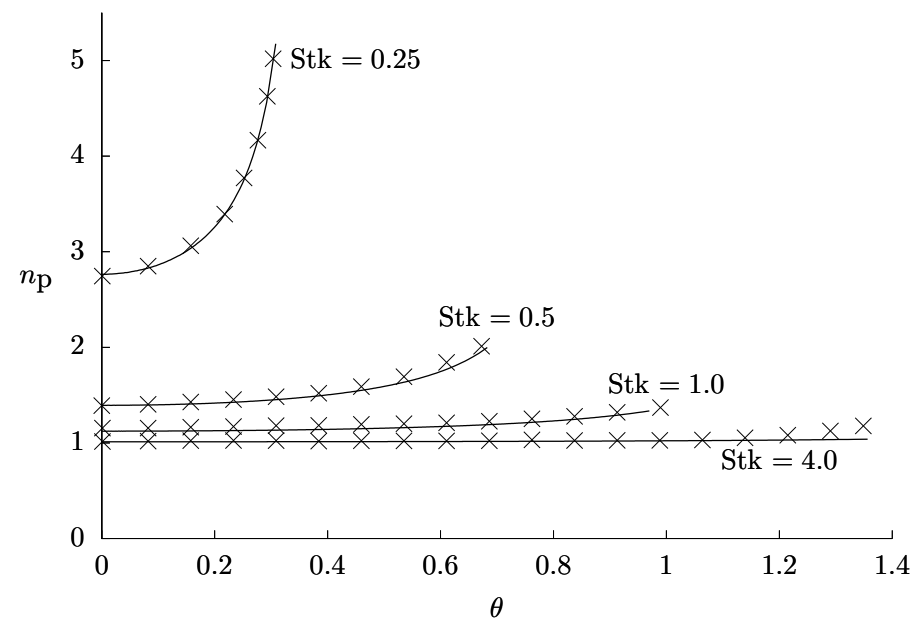

FIG. 1: Number densities of particles deposited on the cylinder surface, inferred from calculations based on the customised version of ANSYS Fluent (solid curves) and given by Wessel and Righi (1988) (symbols).

Note that particle tracking models of ANSYS Fluent were assessed by Greifzu et al. 
(2016). A good agreement between ANSYS Fluent predictions and experimental results was observed.

\section{DILUTE GAS-PARTICLE FLOW AROUND A CYLINDER}

Consider an isothermal, dilute gas-particle flow around an infinitely long circular cylinder. The carrier phase is modelled as a laminar, incompressible, viscous flow of gas (air). The volume fraction and mass loading of particles are assumed to be negligibly small. Hence, interactions between particles and their effect on the carrier phase are ignored. The geometry of the domain and the mesh used in the calculation of the flow around a cylinder are shown in Fig. 2.

Steady-state flow with three Reynolds numbers $(\operatorname{Re}=1,10,100)$ and a transient flow with $\operatorname{Re}=200$ were considered. For these values of Re, the three-dimensional effects of the flow can be ignored (Panton, 1996). The number densities of particles with three Stokes numbers (Stk $=0.05,0.1,0.2$ ) were investigated. Flow velocities and particle diameters corresponding to these Re and Stk for water droplet moving in air around a cylinder with diameter $d_{\mathrm{c}}=0.005 \mathrm{~m}$ are shown in Table 1 . All dimensions in this section are nondimensionalised using the flow velocity and the diameter of the sphere. For the given range of parameters, the diffusion term in Eq. (14) is 12 orders of magnitude smaller than the convection term and does not affect the comparison of the results obtained using EE and FLA.

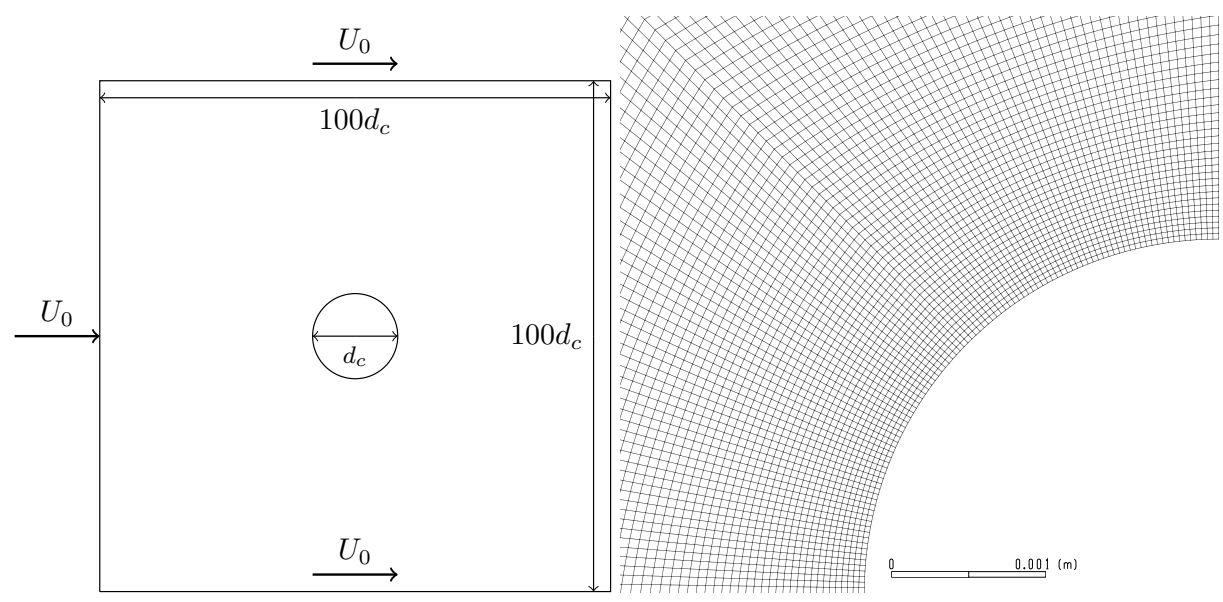

(a)

(b)

FIG. 2: Geometry and a sample of mesh used in the analysis. 
TABLE 1: Flow velocities and particle diameters corresponding to the values of Re and Stk used in the analysis.

\begin{tabular}{ll|cc}
$\operatorname{Re}$ & $U_{0}(\mathrm{~m} / \mathrm{s})$ & Stk & $d_{\mathrm{p}}\left(10^{-6} \mathrm{~m}\right)$ \\
\hline 1 & 0.003 & 0.05 & 164.0 \\
& & 0.1 & 232.0 \\
& & 0.2 & 328.0 \\
\hline 10 & 0.03 & 0.05 & 16.4 \\
& & 0.1 & 23.2 \\
& & 0.2 & 32.8 \\
\hline 100 & 0.3 & 0.05 & 1.64 \\
& & 0.1 & 2.32 \\
& & 0.2 & 3.28
\end{tabular}

\subsection{Computational setup}

A domain $100 d_{\mathrm{c}} \times 100 d_{\mathrm{c}}$ was chosen to minimise the influence of b oundaries of the enclosure on the flow field in the vicinity of the cylinder. A grid independence study was carried out using three meshes with $4 \times 10^{4}, 10^{5}$ and $2.25 \times 10^{5}$ cells whilst monitoring flow v elocity c omponents $n$ ear the $c$ ylinder. The differences in the maximal number densities in all the cases obtained for the meshes with $10^{5}$ and $2.25 \times 10^{5}$ cells were less than $3 \%$. The mesh with $10^{5}$ cells was used for computations. Cell areas varied from $5.76 \times 10^{-5}$ nondimensional units near the cylinder to 1 in the far field.

For the carrier phase, the velocity $\mathbf{u}=\mathbf{U}_{0}$ was set at the inlets (left, top and bottom boundaries); the gauge pressure $p=0$ was set on the right-hand side of the domain. The boundary conditions for the carrier phase velocity at the inlets imply that the flow away from the cylinder is uniform. Eqs. (1) were solved in ANSYS Fluent using the SIMPLE algorithm with a third order MUSCL spatial discretisation and the second order implicit temporal discretisation. In the steady-state case, an absolute iterative convergence of $10^{-15}$ was reached for the discretised Eq. (1). In the transient case, an absolute iterative convergence of $10^{-8}$ for the discretised Eq. (1) was obtained at each time step. A fully developed flow with a Karman vortex sheet occurred after 30 units of simulation time (relative to the time unit $d_{\mathrm{c}} / U_{0}$ ).

In the Eulerian approach, $n_{\mathrm{p}}=1$ was set at the inlets. A zero number density was set on the cylinder surface; the cylinder was assumed to act as a perfect sink for particles. At the outlet, the normal derivative of $n_{\mathrm{p}}$ was set to zero.

In the fully Lagrangian approach, the initial particle number density was set to $n_{0}=$ 1. The components of the Jacobi matrix were set $\left(J_{11}\right)_{0}=\left(J_{22}\right)_{0}=1,\left(J_{12}\right)_{0}=$ $\left(J_{21}\right)_{0}=0$. Assuming that the particle velocity slip at the boundaries is zero, we have $\left(\omega_{11}\right)_{0}=\left(\omega_{12}\right)_{0}=\left(\omega_{21}\right)_{0}=\left(\omega_{22}\right)_{0}=0 . \tau_{\mathrm{f}}$ in expression (7) was taken equal to $U_{0} / d_{\mathrm{c}}$. The starting positions of the particles $\left(x_{0}, y_{0}\right)$ were uniformly distributed on 


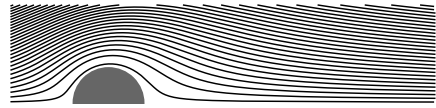

(a) $\operatorname{Re}=1$

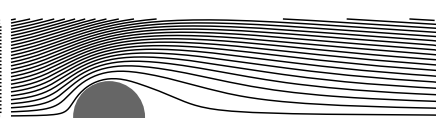

(b) $\operatorname{Re}=10$

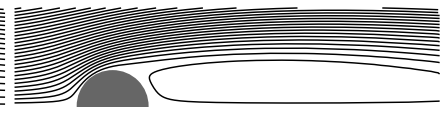

(c) $\operatorname{Re}=100$

FIG. 3: Streamlines obtained for three flow Reynolds numbers.

the left boundary $\left(x_{0}=-50 d_{\mathrm{c}}\right)$ in the projection of the cylinder on the inlet plane: $-d_{\mathrm{c}} / 2 \leq y_{0} \leq d_{\mathrm{c}} / 2$. In the steady-state case, the trajectories of 2000 particles were calculated. In the transient case, 200 particles were injected every 0.6 nondimensional time units.

\subsection{Steady-state flow}

The carrier phase streamlines are shown in Fig. 3. The contours of number density fields are presented in Fig. 4. The top halves of each plot illustrate the number densities obtained using the fully Lagrangian approach; the bottom halves are those obtained using the Eulerian approach. The results obtained using both methods were interpolated over the same Cartesian grid.

As one can see in Figs. 4(a-c), the results predicted using both methods are close for $\mathrm{Stk}=0.05$. For larger values of Stokes number $(\mathrm{Stk}=0.1,0.2)$, the contours predicted by both approaches become visibly different as can be seen in Fig. 4(e-i).

To assess the difference between these contours, the predicted values of particle number density along the axis $y=0$ in front of the cylinder are shown in Fig. 5. Comparing Figs. 4 and 5, one can see that despite different contour shapes predicted by the Eulerian and fully Lagrangian methods, the distributions of predicted particle number densities turned out to be close for some of the combinations of Stk and Re, e.g. Re $=10$, Stk $=0.1$ and $\operatorname{Re}=1$, Stk $=0.2$ (see Fig. 4(e, g) and Fig. 5(a, b)). The relative differences in those cases did not exceed about $4 \%$.

The differences in predicted number densities, using the above mentioned approaches, can be clearly seen for $\mathrm{Re}=10, \mathrm{Stk}=0.2$ and $\mathrm{Re}=100, \mathrm{Stk}=0.1,0.2$. In the case of $\operatorname{Re}=10$, Stk $=0.2$, the fully Lagrangian approach predicts higher values of the gradients of particle number densities close to the cylinder surface compared with the ones predicted by the Eulerian approach. The maximal relative difference in these number densities was almost $17 \%(16.77 \%)$.

For $\mathrm{Re}=100$ and Stk $=0.1,0.2$ (Fig. 5(c)) the maximal relative difference in number densities was $11.1 \%$ and $50.6 \%$, respectively. In these cases the distributions of the particle number densities predicted by the fully Lagrangian approach turned out to be much weaker functions of Stk compared with those predicted by the Eulerian approach. This is related to the fact that the inertial Lagrangian particles are deposited to the cylinder before their velocities and, consequently, number densities change due to 


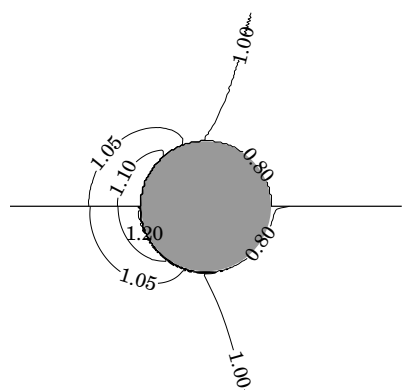

(a) $\mathrm{Stk}=0.05, \operatorname{Re}=1$

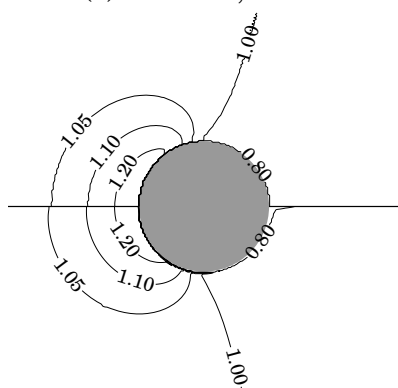

(d) $\operatorname{Stk}=0.1, \operatorname{Re}=1$

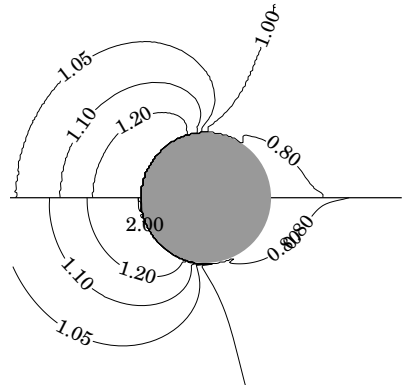

(g) $\operatorname{Stk}=0.2, \operatorname{Re}=1$

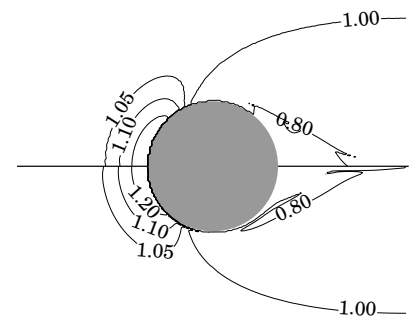

(b) Stk $=0.05, \operatorname{Re}=10$

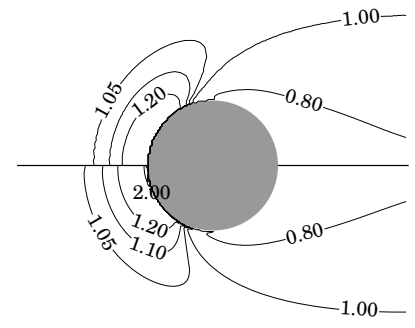

(e) $\mathrm{Stk}=0.1, \mathrm{Re}=10$

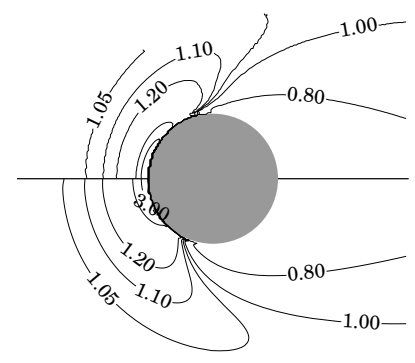

(h) Stk $=0.2, \operatorname{Re}=10$

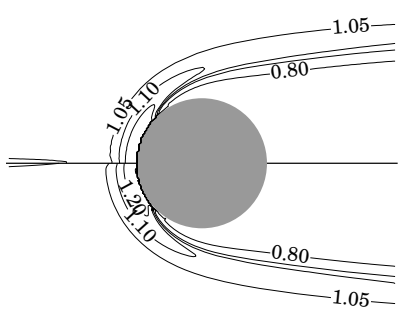

(c) Stk $=0.05, \operatorname{Re}=100$

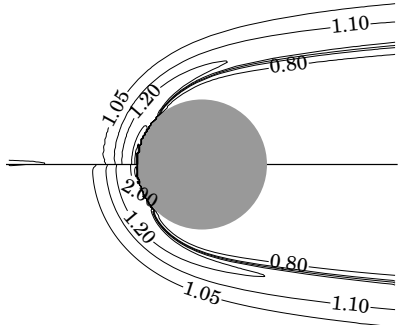

(f) $\operatorname{Stk}=0.1, \operatorname{Re}=100$

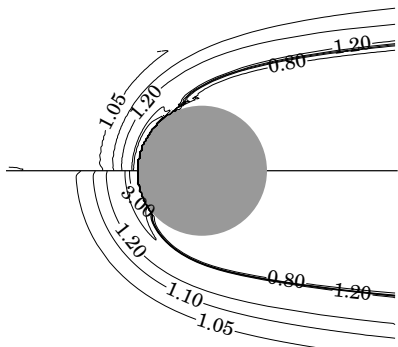

(i) $\mathrm{Stk}=0.2, \operatorname{Re}=100$

FIG. 4: Contours of particle number densities predicted by the fully Lagrangian (top halves) and the Eulerian (bottom halves) methods for a steady-state flow around a cylinder.

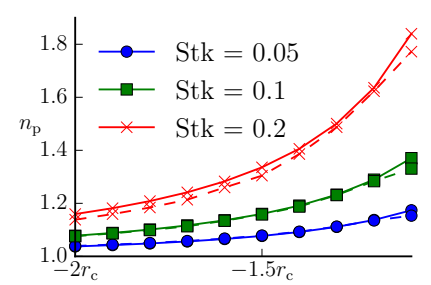

(a) $\operatorname{Re}=1$

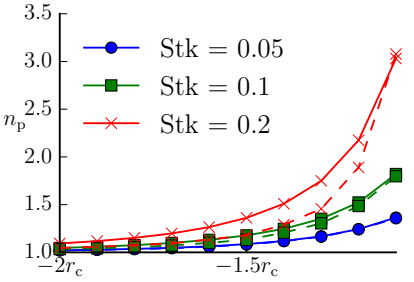

(b) $\operatorname{Re}=10$

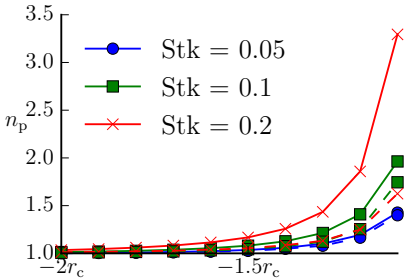

(c) $\operatorname{Re}=100$

FIG. 5: Particle number densities along $x$ axis $(y=0)$ in front of the cylinder predicted by the fully Lagrangian (dashed curves) and Eulerian (solid curves) approaches. 
the changes in the carrier field in front of the cylinder. The EE approach does not capture such behaviour of particles.

\subsection{Transient flow}

The distributions of particle number densities predicted using the above mentioned approaches for $t=48$ nondimensional time units are shown in Fig. 6. The contours of $n_{\mathrm{p}}$, predicted by the Eulerian approach, are shown in Figs. $6(\mathrm{a}, \mathrm{c}, \mathrm{e})$. The distribution of the values of $n_{\mathrm{p}}$, predicted by the fully Lagrangian approach, is shown in Figs. 6(b, $\mathrm{d}, \mathrm{f})$. In the latter figures, solid contours show the distribution of vorticity of the carrier phase and the dots show the values of $n_{\mathrm{p}}$. The locations where the predicted values of $n_{\mathrm{p}}$ are between 1 and 10 times greater than the maximal number density predicted by the Eulerian approach $n_{\max }^{\mathrm{Eul}}$ are shown as crosses $\left(n_{\max }^{\mathrm{Eul}} \leq n_{\mathrm{p}} \leq 10 \cdot n_{\max }^{\mathrm{Eul}}, n_{\max }^{\mathrm{Eul}}=3.5\right)$. The locations where the predicted values of $n_{\mathrm{p}}$ are more than 10 times greater than the maximal number density predicted by the Eulerian approach $\left(10 \cdot n_{\max }^{\mathrm{Eul}} \leq n_{\mathrm{p}}\right)$ are shown as diamonds.

As follows from our analysis, the Eulerian approach predicts preferential accumulation of particles in the regions of low vorticity in agreement with previously reported results (Ferry and Balachandar, 2001; Maxey, 1987). A similar behaviour is predicted by the fully Lagrangian approach. The plots of the relative numbers of particles located in the regions with given vorticity versus the values of vorticity are shown in Fig. 7 for three values of Stk (the areas under the curves are equal to 1 ). These numbers were counted behind the cylinder and averaged over 16 cycles of vortex shedding. This effect is relatively weak at lower Stokes numbers ( $\mathrm{Stk}=0.05,0.1$ ), but becomes stronger for Stk $=0.2$. The preferential accumulation of particles in the region of low vorticity is consistent with the results presented in Figs. 6(b, d, f), which show that particle number densities have local maxima between the regions of high vorticity (see the locations of crosses and diamonds in these figures). The trajectories intersect between those regions.

The maximal values of particle number densities predicted by both methods for three values of Stk are shown in Table 2. In all cases the Eulerian approach (EE) predicts the highest values of $n_{\mathrm{p}}$ in the stagnation point in front of the cylinder. In contrast to the Eulerian approach, the fully Lagrangian approach always predicted the highest number densities behind the cylinder, where trajectory intersections occurred. For Stk $\geq 0.1$ the values of $n_{\mathrm{p}}$ predicted by the fully Lagrangian approach are several orders of magnitude higher than those predicted by the Eulerian approach. Note that in the regions of high particle number density, the particles might interact with each other and the assumptions of both methods, that this interaction can be ignored, are no longer valid. The fully Lagrangian approach allows us to identify such regions, while the Eulerian method does not give us any indication of the trajectory intersections. 


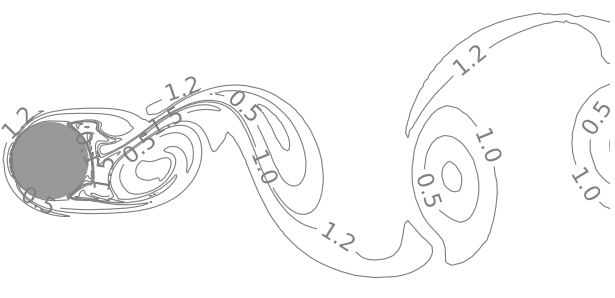

(a) $\mathrm{Stk}=0.05$

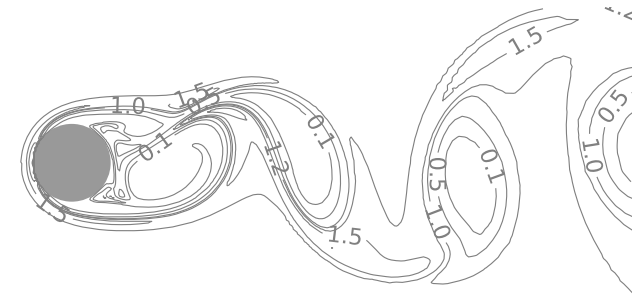

(c) $\mathrm{Stk}=0.1$

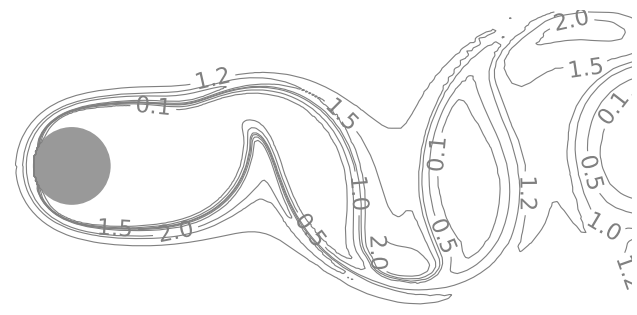

(e) $\mathrm{Stk}=0.2$

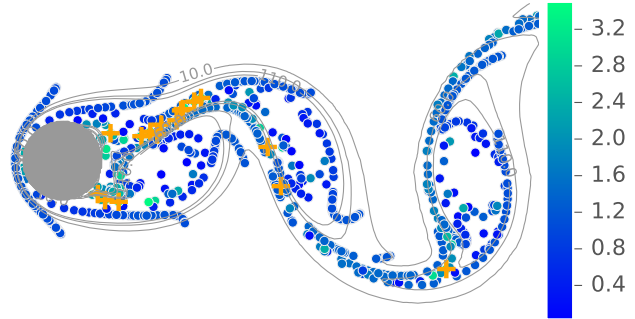

(b) Stk $=0.05$

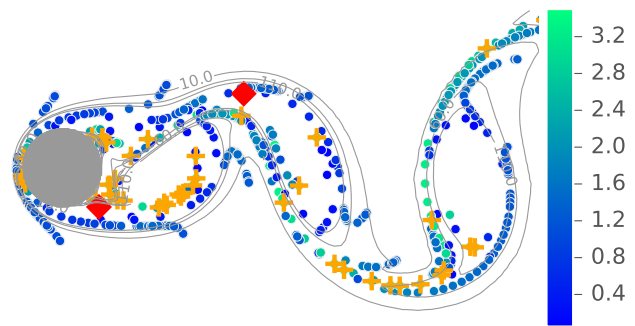

(d) $\operatorname{Stk}=0.1$

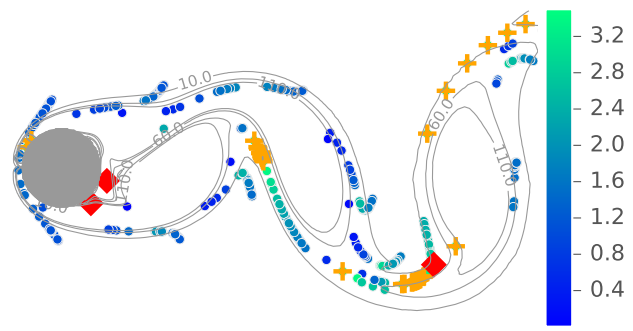

(f) $\mathrm{Stk}=0.2$

FIG. 6: Contours of particle number densities predicted by the Eulerian approach for three values of Stk (a, c, e); contours of vorticity of the carrier phase and particle number densities predicted for three values of Stk by the fully Lagrangian approach $(b, d$, f). The results refer to the transient flow around a cylinder with $\operatorname{Re}=200$ at $t=48$ nondimensional time units. The locations where the predicted values of $n_{\mathrm{p}}$ are between 1 and 10 times greater than the maximal number density predicted by the Eulerian approach $n_{\text {max }}^{\text {Eul }}$ are shown as crosses $\left(n_{\max }^{\mathrm{Eul}} \leq n_{\mathrm{p}} \leq 10 \cdot n_{\max }^{\mathrm{Eul}}, n_{\max }^{\mathrm{Eul}}=3.5\right)$. The locations where the predicted values of $n_{\mathrm{p}}$ are more than 10 times greater than the maximal number density predicted by the Eulerian approach $\left(10 \cdot n_{\max }^{\text {Eul }} \leq n_{\mathrm{p}}\right)$ are shown as diamonds. The location of the points where these number densities are less than $n_{\max }^{\mathrm{Eul}}$ are shown as circles in the range of colours from blue to green.

\section{DROPLET DYNAMICS IN A GASOLINE FUEL SPRAY}

The formation and dynamics of fuel sprays are complex processes involving cavitation inside the nozzle, development of a jet, conversion of the jet into a liquid sheet and ligaments, primary and secondary break-up modes (Heywood, 1988; Sazhin, 2014). Faithf 


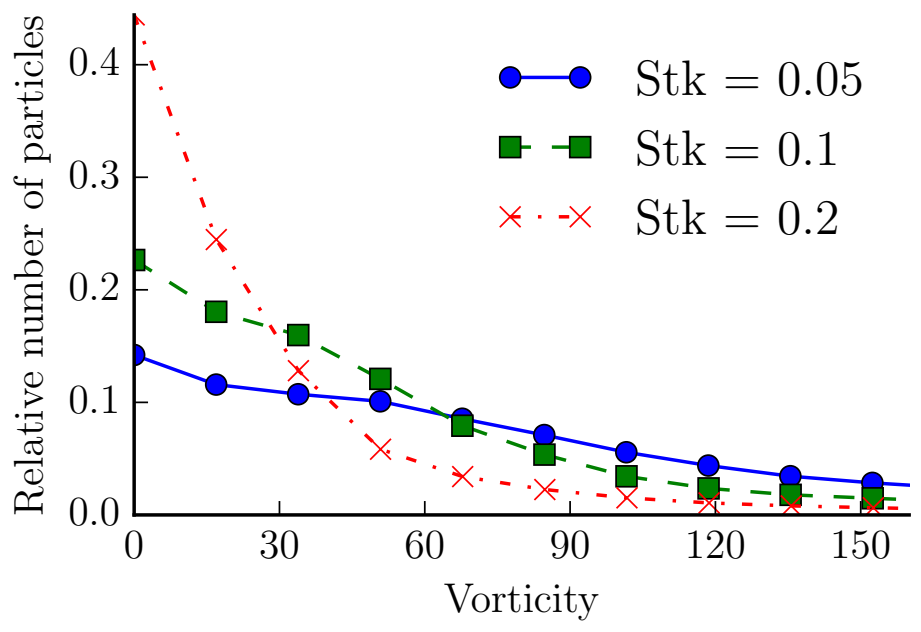

FIG. 7: Relative numbers of particles versus the vorticity of the carrier phase in the locations of particles.

TABLE 2: Maximal values of number densities $n_{\mathrm{p}}$ predicted by the Eulerian approach (EE) and the fully Lagrangian approach (FLA).

\begin{tabular}{l|ccc} 
Stk & 0.05 & 0.1 & 0.2 \\
\hline EE & 2.66 & 6.93 & 23.0 \\
FLA & 14.9 & 466.0 & $9.01 \times 10^{5}$
\end{tabular}

modelling of these processes is a challenging task. In the simulation of a hollow-cone gasoline spray in an internal combustion engine, Hermann et al. (2008) used the internal nozzle flow calculation to define the initial conditions (Cauchy problem) for the transient simulation of the mixture formation. Gavaises et al. (2006) went further and simulated not only the internal nozzle flow, but also the liquid sheet in the near-nozzle region. The velocity field for the carrier phase, calculated using the Eulerian approach, was then used as an input to the Lagrangian spray simulation.

Both Hermann et al. (2008) and Gavaises et al. (2006) demonstrated, that the initial injection process has a strong influence on the formation of the fully-developed spray. On the other hand, it was shown in (Iyer et al., 2004; Takagi and Moriyoshi, 2004) that an Eulerian-Lagrangian multi-phase spray simulation, with carefully selected initial conditions, can give a good prediction of such hollow-cone spray characteristics as spray cone angle, tip penetration and outer vortex height.

It is not the intention to perform a detailed simulation of droplet dynamics in gasoline fuel sprays. Rather, the analysis presented here will be restricted to the investigation 
of the applicability of the new customised version of ANSYS Fluent, using a fully Lagrangian approach, to the investigation of some features of the droplet dynamics.

\subsection{Experimental setup}

The characteristics of the injection process of a gasoline fuel spray were experimentally investigated. A modern piezoelectric fuel injector with direct needle actuation, commonly reported in spray-guided, gasoline combustion systems, was selected for the study. During fuel injection, the outwardly opening, pintle type needle produces an initially thin liquid sheet that forms into a hollow-cone spray comprising of dispersed droplets (Hermann et al., 2008). In this study, the fuel injector was mounted in a vertical position at the top of an enclosed square glass chamber ( $200 \mathrm{~mm}$ by $200 \mathrm{~mm}$ ) of height $250 \mathrm{~mm}$. The ambient gas temperature and pressure were $293 \mathrm{~K}$ and 1 bar respectively. Gasoline was substituted with a surrogate primary reference fuel $(2,2,4$,Trimethylpentane). The fuel injection pressure was 100 bar. The injection duration was $1 \mathrm{~ms}$ and the time between consecutive injections was $1 \mathrm{~s}$. The liquid mass flow rate profile was determined using an injection rate tube. Several experimental techniques were used to characterise the spray. High-speed photography was used to capture the spray penetration rate and evolution of the spray shape following injection. In addition, a Dantec Dynamics, Classical, two-component phase Doppler anemometer (PDA) was used to measure the droplet size and velocity distributions at a series of grid locations. The experimental set-up is illustrated in Fig. 8. The effects of droplet heating and evaporation have been ignored in our analysis.

\subsection{Computational setup}

The Eulerian-Lagrangian model, used for identification of particle trajectories, was adjusted to account for the conditions of the gasoline injection through an outwardly opening pintle injector. We consider a two-dimensional axisymmetric laminar flow of a compressible fluid. The momentum exchange between the $\mathrm{c}$ arrier and dispersed phases is taken into account (two-way coupling). The details of the spray formation are not considered and it is assumed that droplets are injected close to the nozzle exit. The droplet sizes distribution was taken from the experimental observations. Initial velocities of droplets were estimated from the liquid mass flow rate.

The effects of turbulent diffusion, droplet break-up and interaction between droplets in the high concentration region near the nozzle exit are ignored. Also we assume that the Stokes drag law is valid. This approach will not provide an accurate description of the details of the spray formation near the nozzle (where experimental uncertainty is greatest), but it is expected to give a qualitative description of the evolution of a droplet cloud at large distances from the nozzle, consistent with the scales of the combustion

chamber size. Whilst the spray model in this case may be considered to lack technical 


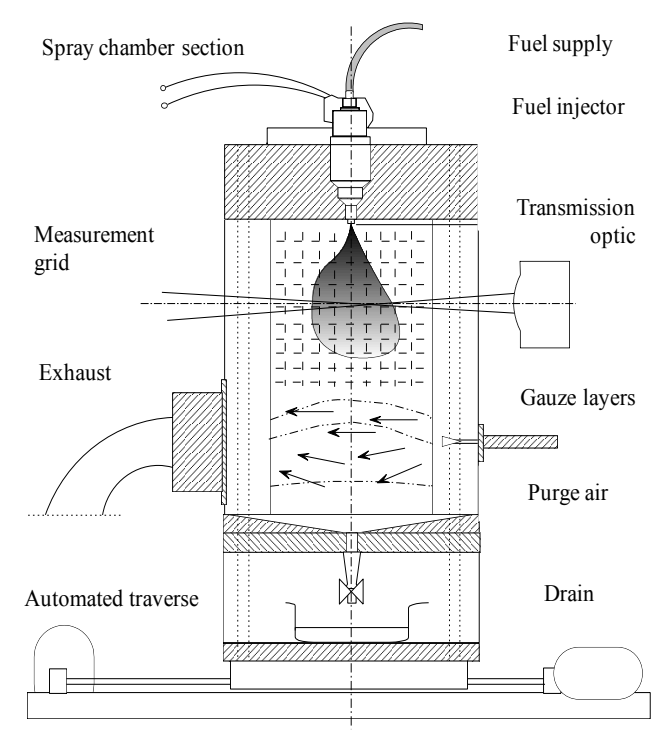

FIG. 8: Experimental setup

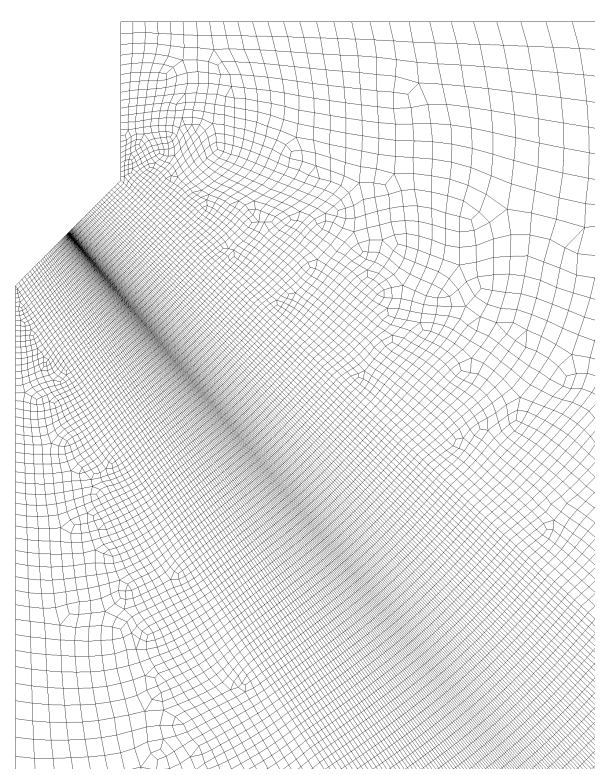

FIG. 9: Computational mesh near the nozzle

depth, it still provides a good demonstration of the potential capabilities of the fully Lagrangian method for the analysis of droplet number densities.

The Navier-Stokes equations for the carrier phase were solved in ANSYS Fluent using the density based solver with implicit Roe-FDS flux type, a third order MUSCL spatial discretisation and a second order implicit temporal discretisation. The region of computational mesh near the nozzle is shown in Fig. 9. During the injection period $\left(10^{-4} \mathrm{~s}\right) 20$ droplets of various sizes were injected with a velocity of $150 \mathrm{~m} \mathrm{~s}^{-1}$ at each flow time step $\left(5 \times 10^{-7} \mathrm{~s}\right)$. In total, the trajectories of 20000 droplets were calculated. Droplet diameters $d_{\mathrm{p}}$ were distributed between $1 \mu \mathrm{m}$ and $30 \mu \mathrm{m}$ with the mean value $10 \mu \mathrm{m}$ using the Rosin-Rammler function with the spread parameter equal to 3.5.

\subsection{Results}

The time evolution of droplets, predicted by the ANSYS Fluent, overlayed on the images of an experimentally observed spray, are shown in Fig. 10. The spray was illuminated from the right-hand side. The injector tip is located in the upper left corner of the images. The dimensions of the injector tip are used as the spatial scaling factor for the comparison of the experimental and numerical results. Time was measured from the start of the injection, and time scales are kept the same. As we can see in Figs. 10(b-d), the observed vortices are correctly predicted by our simulations, both from the point of view of timing and location. The directions of rotation of vortices predicted by numerical simulations 
and inferred from experimental data (videos) were the same. This result agrees with the earlier results presented by Hermann et al. (2008). Thus, we consider the evolution of the droplets cloud in the area of big vortex formation to qualitatively agree with the experimental observation.

The trajectories of the droplets of three size classes, calculated during $5 \times 10^{-3} \mathrm{~s}$ after the start of the injection, are shown in Fig. 11. Only 50 representative trajectories are selected for each droplet size and only one in 100 points in each trajectory is presented. As one can see from this figure, larger droplets $\left(d_{\mathrm{p}}=10 \mu \mathrm{m}, d_{\mathrm{p}}=20 \mu \mathrm{m}\right.$, see Figs. 11(b-c)) are moving almost along straight lines until the point $y=0.04 \mathrm{~m}$, where trajectories deviate from the straight line. This is the region, where a large vortex is formed after the end of the injection (see Fig. 10(c)). Due to the low inertia, the smaller droplet $\left(d_{\mathrm{p}}=2 \mu \mathrm{m}\right.$, see Fig. 11(a)) trajectories deviate from a straight line at approximately $y=0.03 \mathrm{~m}$. Due to the interaction of droplets with small vortices, the shapes of these trajectories are more complex, with a number of turns and loops. Entrained by the reverse flow along the axis of symmetry, these droplets reach areas above the injector tip. This is not observed with the larger droplets. Such behaviour is consistent with experimental observations.

Comparing Figs. 10 and 11 we can see, that despite the deviation of trajectories of individual droplets from the straight line, the tip of the spray stays coherent. This is related to the fact that when the droplets at the tip lose their momentum, the droplets following their trail are subjected to experiencing less air resistance and form a new tip.

As mentioned in the Introduction, the main advantage of the fully Lagrangian method used in our study over the conventional Lagrangian approach is that it allows us to predict not only the positions of the droplets, but also their number densities. The evolution of $n_{\mathrm{p}}$ along the trajectories is shown in Fig. 11. For better visualisation of the results, the number densities $n_{\mathrm{p}} \geq 10$ were truncated to 10 .

Number densities of the droplets with $d_{\mathrm{p}}=10 \mu \mathrm{m}$ and $d_{\mathrm{p}}=20 \mu \mathrm{m}$ (Figs. 11(b-c)) were mainly in the range $0 \leq n_{\mathrm{p}} \leq 10$. Note that $n_{\mathrm{p}}$ decreased along some trajectories. The number densities of droplets with $d_{\mathrm{p}}=2 \mu \mathrm{m}$ (Fig. 11(a)) can be very high in some regions (regions of droplet accumulation). Also, we can see from Fig. 11, that some of the small droplet trajectories include the sections with sudden colour change. This indicates a change in $n_{\mathrm{p}}$.

To explain this phenomenon, we present some selected trajectories in Fig. 12. In this figure, the trajectory points are highlighted according to the sign of the Jacobians determinant: " $\times$ " refers to its positive values, while "o" refers to its negative values. Changes in sign indicate zero values of the determinant when the number density of droplets is infinitely large (Eq. (8)). As mentioned earlier, these singularity points appear due to trajectory intersection or folds (Rybdylova et al., 2015). As one can see in Fig. 12, the trajectories of droplets with $d_{\mathrm{p}}=2 \mu \mathrm{m}$ have more singularity points, than those of droplets with $d_{\mathrm{p}}=10 \mu \mathrm{m}$ and $d_{\mathrm{p}}=20 \mu \mathrm{m}$. Note that in Fig. 12 we see that folds can occur even if trajectories are almost straight. 


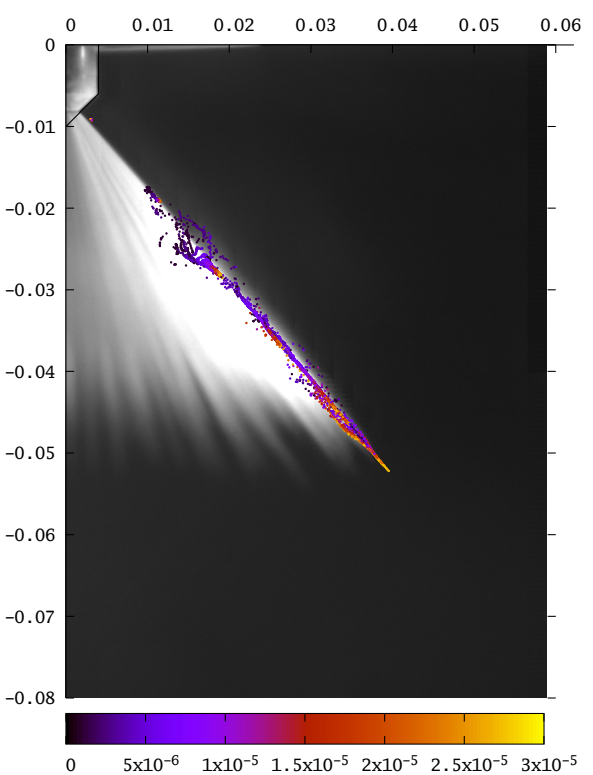

(a) $t=0.5 \times 10^{-3}$

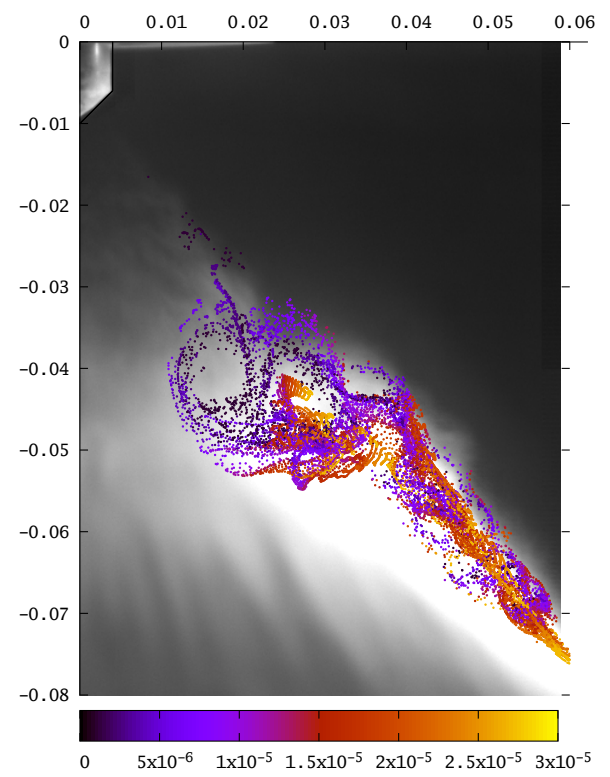

(c) $t=1.5 \times 10^{-3}$

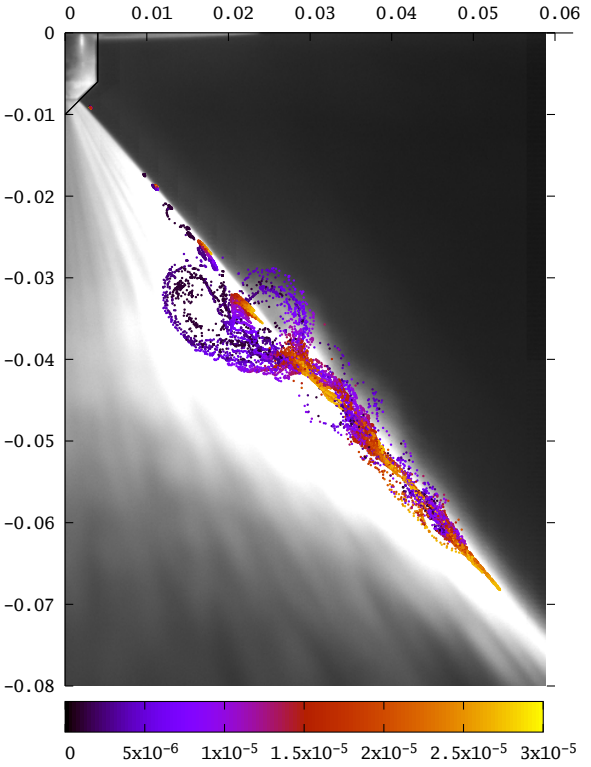

(b) $t=1 \times 10^{-3}$

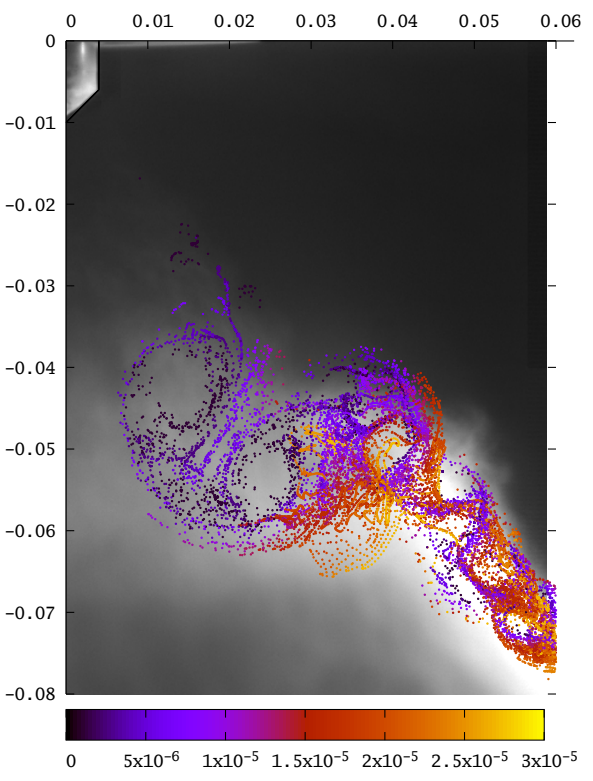

(d) $t=2 \times 10^{-3}$

FIG. 10: The time evolution of droplets with diameters between $10^{-6} \mathrm{~m}$ and $30 \times$ $10^{-6} \mathrm{~m}$ shown by colour, as predicted by the ANSYS Fluent, overlayed on the images of an experimentally observed spray. 
Note that due to the rapid changes in the velocity and the length scales of the injected flow (both in time and space), the diameters of the droplets cannot be associated with constant Stokes numbers.

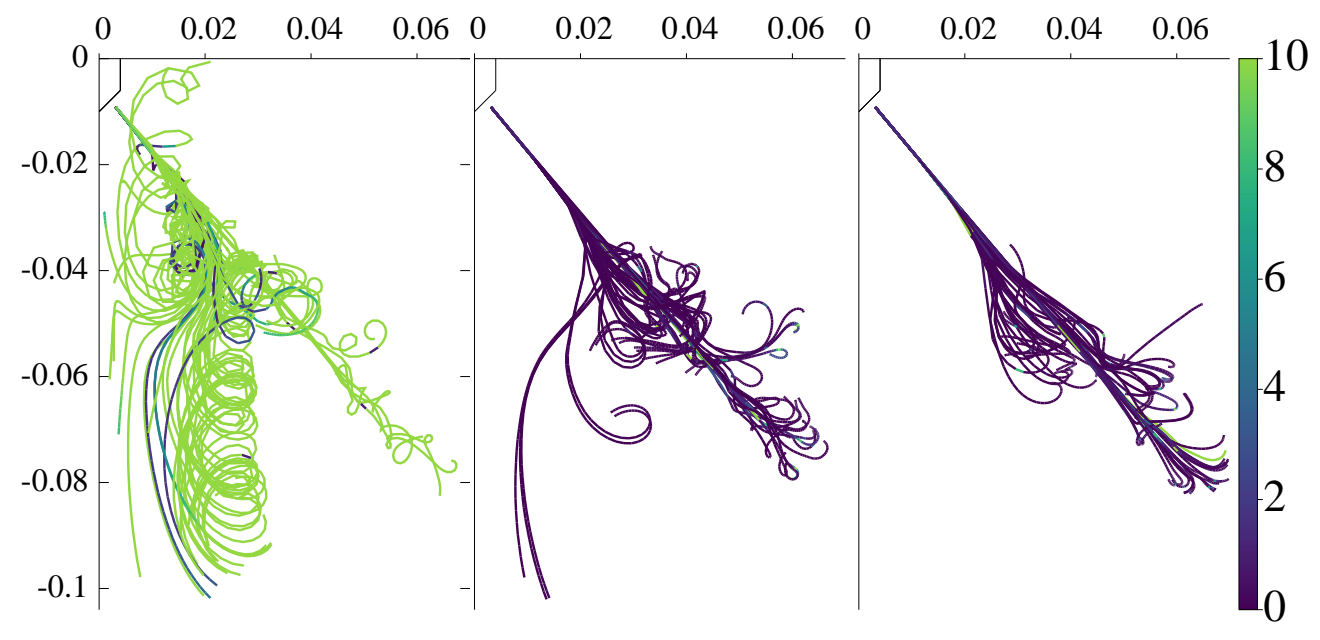
(a) $d_{\mathrm{p}}=2 \mu \mathrm{m}$
(b) $d_{\mathrm{p}}=10 \mu \mathrm{m}$
(c) $d_{\mathrm{p}}=20 \mu \mathrm{m}$

FIG. 11: Trajectories of droplets of three sizes during the first $5 \times 10^{-3} \mathrm{~s}$ after the start of injection. Colours show number densities of droplets, all values above 10 are truncated to 10 .

\section{CONCLUSIONS}

Two approaches (equilibrium Eulerian and fully Lagrangian) to calculating the number densities of inertial particles in dilute gas-particle flows have been implemented into the CFD code ANSYS Fluent using User Defined Functions. A new customised version of ANSYS Fluent has been used to model two distinct cases: dilute gas-particle flow around an infinite cylinder, and a hollow-cone gasoline fuel spray.

For a gas-particle flow around a stationary cylinder, number densities of particles with Stokes numbers 0.05, 0.1, 0.2 in steady-state and transient laminar flows at four Reynolds numbers ( $\operatorname{Re}=1,10,100$ and 200) have been predicted. In a steady-state case, flows with Reynolds numbers 1, 10, and 100 have been investigated. It has been shown that the results predicted using both equilibrium Eulerian and fully Lagrangian methods are almost identical at small Stokes and small Reynolds numbers (Stk $=0.05$, $\operatorname{Re}=1$ ). For larger values of these numbers (Stk $=0.1,0.2$; $R e=10,100)$, the fully Lagrangian approach predicts higher values of the gradients of particle number densities in front of the cylinder compared with the ones predicted by the Eulerian approach.

The distribution of particle number densities in a transient flow around a cylinder 


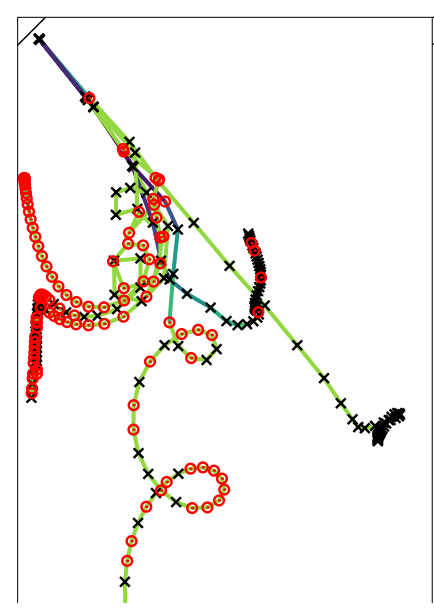

(a) $d_{\mathrm{p}}=2 \mu \mathrm{m}$

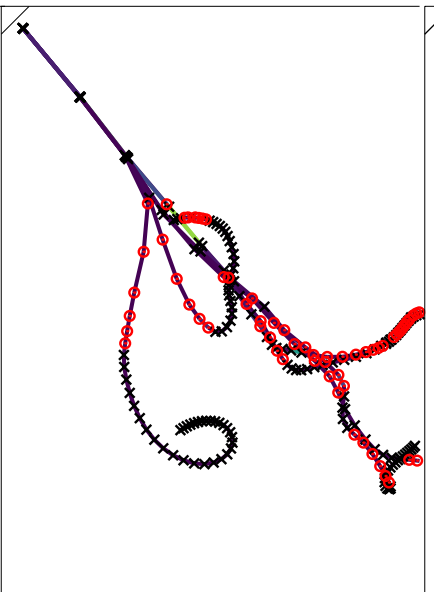

(b) $d_{\mathrm{p}}=10 \mu \mathrm{m}$

(c) $d_{\mathrm{p}}=20 \mu \mathrm{m}$

FIG. 12: Trajectories of droplets of three sizes during the first $5 \times 10^{-3} \mathrm{~s}$ after the start of injection. Line colours show number densities of droplets, all values above 10 are truncated to 10. Trajectory points are marked according to the sign of the Jacobian determinants: " $\times$ " correspond to positive values, and "o" correspond to negative values.

with Reynolds number equal to 200 have been investigated. Both equilibrium Eulerian and the fully Lagrangian methods predict high values of these densities between the regions of high vorticity and very low values in the vortex cores, in agreement with the earlier reported results. It has been shown that the values obtained using the Eulerian approach do not capture local accumulation zones predicted by the fully Lagrangian method. In addition, the Eulerian method cannot predict correctly both the locations and the magnitude of the maxima of particle number densities. For Stk $\geq 0.1$ the values predicted by the fully Lagrangian approach are shown to be several orders of magnitude higher than those predicted by the Eulerian approach. It is noted that in the regions of infinitely high particle number density, the particles are expected to interact with each other and the assumption that this interaction can be ignored is no longer valid. The fully Lagrangian approach allows us to predict the occurrence of such regions, while the Eulerian method does not give us any indication of the intersections of the trajectories.

The customised version of ANSYS Fluent, with fully Lagrangian approach (FLA), implemented into it, has been applied to the calculate the number densities of droplets in a direct injection type gasoline spray. Results show good qualitative agreement between the numerical simulation and experimental observations. The flow reversal, observed close to the nozzle, and vortex-like structures, developed along the inner and outer boundaries of the hollow-spray cone, are correctly reproduced by the simulations. It is shown, that droplets with $d_{\mathrm{p}}=2 \mu \mathrm{m}$ produce a greater number of accumulation regions 
with very high number densities, while number densities of droplets with $d_{\mathrm{p}}=10 \mu \mathrm{m}$ and $d_{\mathrm{p}}=20 \mu \mathrm{m}$ decrease in some regions.

The results of the study are believed to be pertinent to modelling of fuel sprays in direct injection gasoline combustion systems where there is a need to accurately model the concentration of the air-fuel mixture, especially during part-load, lean, stratifiedcharge, operating conditions. The predictions of the size, concentration and trajectory of fuel droplets in the vortex-ring like structures, in the vicinity of the spark gap are believed to be of paramount importance to the optimisation of the mixture preparation processes.

\section{ACKNOWLEDGMENTS}

The authors are grateful to the EPSRC (UK) (grants EP/K005758/1 and EP/M002608/1), Royal Society (UK) and the Russian Foundation for Basic Research (grants No. 15-0106135 and No. 16-51-10024) for their financial support.

\section{REFERENCES}

Cash, J. R. and Karp, A. H., A variable order runge-kutta method for initial value problems with rapidly varying right-hand sides, ACM Trans. Math. Softw., vol. 16, no. 3, pp. 201-222, 1990.

Fernandez de la Mora, J. and Rosner, D. E., Inertial deposition of particles revisited and extended: Eulerian approach to a traditionally lagrangian problem, Physicochem. Hydrodyn., vol. 2, pp. 1-21, 1981.

Ferry, J. and Balachandar, S., A fast eulerian method for disperse two-phase flow, International Journal of Multiphase Flow, vol. 27, no. 7, pp. 1199-1226, 2001.

Friedlander, S. K., Smoke, Dust, and Haze: Fundamentals of Aerosol Dynamics, Topics in chemical engineering, Oxford University Press, 2000.

Gavaises, M., Tonini, S., Marchi, A., Theodorakakos, A., Bouris, D., and Matteucci, L., Modelling of internal and near-nozzle flow of a pintle-type outwards-opening gasoline piezoinjector, International Journal of Engine Research, vol. 7, no. 5, pp. 381-397, 2006.

Greifzu, F., Kratzsch, C., Forgber, T., Lindner, F., and Schwarze, R., Assessment of particletracking models for dispersed particle-laden flows implemented in openfoam and ansys fluent, Engineering Applications of Computational Fluid Mechanics, vol. 10, no. 1, pp. 30-43, 2016.

Healy, D. P. and Young, J. B., Full lagrangian methods for calculating particle concentration fields in dilute gas-particle flows, Proceedings of the Royal Society of London A: Mathematical, Physical and Engineering Sciences, vol. 461, no. 2059, pp. 2197-2225, 2005.

Hermann, A., Kruger, C., Schaupp, U., Arndt, S., Gartung, K., Hubel, M., Spicher, U., and Velji, A., Numerical and diagnostic analysis of spray propagation and vortex formation of piezoa-injectors and their sensitivity to the internal nozzle flow, Proceedings / 8th International Symposium on Combustion Diagnostics, Mainz-Kastel AVL Deutschland GmbH, pp. 471486, 2008.

Heywood, J., Internal Combustion Engine Fundamentals, McGraw-Hill Education, 1988. 
Iyer, C., Han, Z., and Yi, J., Cfd modeling of a vortex induced stratification combustion (visc) system, SAE Technical Paper, 2004.

Kolakaluri, R., Subramaniam, S., and Panchagnula, M., Trends in multiphase modeling and simulation of sprays, International Journal of Spray and Combustion Dynamics, vol. 6, no. 4, pp. 317-356, 2014.

Maxey, M. R., The gravitational settling of aerosol particles in homogeneous turbulence and random flow fields, Journal of Fluid Mechanics, vol. 174, pp. 441-465, 1987.

Nigmatulin, R. I., Dynamics Of Multiphase Media, no. 1 in Dynamics of Multiphase Media, CRC Press, 1990.

Osiptsov, A. N., Mathematical modeling of dusty-gas boundary layers, Appl. Mech. Rev., vol. 50, no. 6, pp. 357-370, 1997.

Osiptsov, A. N., Lagrangian modelling of dust admixture in gas flows, Astrophysics and Space Science, vol. 274, no. 1-2, pp. 377-386, 2000.

Panton, R. L., Incompressible Flow, John Wiley \& Sons, Inc., 1996.

Rybdylova, O., Osiptsov, A. N., Sazhin, S. S., Begg, S., and Heikal, M., A fully meshless method for "gas-evaporating droplet" flow modelling, PAMM, vol. 15, no. 1, pp. 685-686, 2015.

Rybdylova, O., Qubeissi, M. A., Braun, M., Crua, C., Manin, J., Pickett, L. M., de Sercey, G., Sazhina, E. M., Sazhin, S. S., and Heikal, M., A model for droplet heating and its implementation into ansys fluent, International Communications in Heat and Mass Transfer, vol. 76, pp. 265-270, 2016.

Sazhin, S. S., Droplets and Sprays, Springer-Verlag London, 2014.

Sazhina, E. M., Sazhin, S. S., Heikal, M. R., Babushok, V. I., and Johns, R. J. R., A detailed modelling of the spray ignition process in diesel engines, Combustion Science and Technology, vol. 160, no. 1, pp. 317-344, 2000.

Takagi, M. and Moriyoshi, Y., Modelling of a hollow-cone spray at different ambient pressures, International Journal of Engine Research, vol. 5, no. 1, pp. 39-52, 2004.

Wessel, R. A. and Righi, J., Generalized correlations for inertial impaction of particles on a circular cylinder, Aerosol Science and Technology, vol. 9, no. 1, pp. 29-60, 1988. 